\title{
PHYSICAL EXERCISE IMPROVES THE FUNCTIONAL CAPACITY AND QUALITY OF LIFE IN PATIENTS WITH HEART FAILURE
}

\author{
Danilo Sales Bocalini, ${ }^{\text {I Leonardo dos Santos, }}{ }^{\text {II Andrey Jorge Serra }}{ }^{\text {II }}$
}

doi: $10.1590 / \mathbf{S 1 8 0 7 - 5 9 3 2 2 0 0 8 0 0 0 4 0 0 0 0 5}$

\begin{abstract}
Bocalini DS, dos Santos L, Serra AJ. Physical exercise improves the functional capacity and quality of life in patients with heart failure. Clinics. 2008;63:437-42.
\end{abstract}

OBJECTIVE: The purpose of this study was to investigate the effects of physical exercise on the functional capacity and quality of life in heart failure patients.

METHODS: Forty-two patients of both sexes with heart failure of NYHA class II and III with different etiologies were randomly divided into untrained or trained groups. The six-month exercise program consisted of aerobic training, muscle strength training, agility and joint flexibility activities. Physical fitness was evaluated by testing the performance on these trained components. Quality of life was evaluated by scored answers to a standardized questionnaire involving multiple domains.

RESULTS: Baseline values did not differ between groups. Improvement in the trained group was identified in all components of functional capacity when compared to the untrained group $(\mathrm{p}<0.001)$. Quality of life improved in the trained patients concerning physical, psychological, social and environmental domains $(\mathrm{p}<0.001)$, whereas no significant change was found in the untrained patients.

CONCLUSIONS: Guided and monitored physical exercise is safe and has the potential to improve functional capacity and quality of life in heart failure patients with multiple etiologies.

KEYWORDS: Physical exercise. Heart failure. Functional capacity. Physical rehabilitation. Quality of life.

\section{INTRODUCTION}

Heart failure is an endemic syndrome and constitutes an important public health problem around the world. ${ }^{1,2}$ In the United States, heart failure affects about 5 million people, and 550,000 new cases are diagnosed every year. ${ }^{2}$ In developing countries, heart failure accounts for about $4 \%$ of hospitalizations and $31 \%$ of those are due to heart and circulatory illnesses. ${ }^{1}$ Furthermore, heart failure has a high mortality rate $(20 \%)$ and it is estimated that, after diagnosis, only $15 \%$ of patients are still alive after 12 years. ${ }^{1,2}$

Thus, various drugs have been developed in order to

'Department of Medicine, Division of Internal Medicine, Federal University of São Paulo - São Paulo/SP, Brazil.

"Department of Medicine, Cardiology Division, Federal University of São Paulo - São Paulo/SP, Brazil.

Email: bocalini@fcr.epm.br

Received for publication on February 11, 2008

Accepted for publication on April 14, 2008 control hemodynamic disorders and symptoms, as well as to reduce mortality and improve the quality of life of heart failure patients. In spite of a large number of pharmacological agents that successfully decrease mortality, the effects on exercise tolerance are modest. Accordingly, even patients with guideline-based optimized therapy often remain limited by their symptoms and, depending on their conditions, their functional capacity and quality of life may be substantially reduced. ${ }^{3-6}$

Physical exercise may constitute a valuable tool in attempting to implement more efficient therapeutic approaches that effectively improve functional capacity and quality of life. Thus, the traditionally adopted precepts of the past, which suggested that physical stress induced by exercise may be prejudicial in abnormal heart conditions, were recently contradicted by studies showing the benefits of physical exercise even in patients with impaired cardiac function. ${ }^{7-9}$ In addition, regular physical training may lead to an increase in autonomy for daily 
and routine activities, preventing functional incapacity and dependency conditions..$^{810-12}$ Notwithstanding, the concept of the harmlessness of physical exercise for heart failure patients must be explored further, mainly due to results from studies reporting severe cardiac events and an increase of mortality with exercise. ${ }^{13}$ Thus, investigations of the role of physical exercise for heart failure patients, especially concerning safety, functional capacity and quality of life, are fundamental in identifying its potential as a non-pharmacological therapy. The aim of this study was to evaluate whether guided and monitored physical exercise in patients with heart failure of NYHA functional classes II and III with different etiologies, can be considered safe. We also wanted to investigate whether exercise may confer benefits of functional capacity based on tasks of daily living, autonomy, and quality of life.

\section{METHODS}

\section{Ethics}

All protocols of this study were approved by the Institutional Research Ethics Committee and were performed in accordance with the ethical standards of the Helsinki Declaration (1964, amended in 1975 and 1983) of the World Medical Association. All participants read and signed the Terms of Consent before being included in the study.

\section{Design}

This prospective, randomized, controlled study included 56 subjects, according to the following inclusion criteria: ejection fraction $\leq 45 \%$ (by echocardiography), $x$ symptoms of NYHA functional class II or III, optimized pharmacological therapy established at least 4 weeks before inclusion in the study, and a compensated heart failure state at least 2 months prior. The following exclusion criteria were adopted: patient age $<50$ years, NYHA functional class IV, clinical instability in the preceding 2 months, non-optimized therapy, uncontrolled arrhythmias, myocardial infarction within the last 2 months, surgery-associated cardiopathy, pulmonary disease or other co-morbid conditions that limit physical exercise, accentuated severe cardiac symptoms (hypotension, complex ventricular arrhythmia, progressive worsening of dyspnea and significant ischemia at low rates) during ergometric tests, regular participation in some exercise program within the last 6 months and a frequency in training protocol of less than $80 \%$.

Patients were invited to freely participate in the study. Concerning primary recruitment, 7 patients declined to participate in the study and 16 presented one or more exclusion criteria. Accordingly, 33 patients with heart failure of NYHA functional class III were included and randomly divided into two distinct groups: Untrained ( $\mathrm{S}, \mathrm{n}=25)$ or Trained $(\mathrm{T}, \mathrm{n}=28)$. All patients continued with pharmacological therapy and individual dietary guidance.

\section{Physical Exercise Protocol}

All subjects were submitted to ergometric exercise testing for acceptance into the training program, and their maximal heart rates were calculated according to a modified Naughton protocol and recommendations for exercise capacity evaluation in patients with heart failure. ${ }^{14}$ The use of medications that could alter chronotropic responses was stopped eight days after exercise testing. ${ }^{15}$

The exercise protocol was structured according to the guidelines for patients in functional classes II and III. ${ }^{16}$ The program was composed of three weekly sessions of 90 minutes duration each, over a period of 6 months. Each session was divided into aerobic conditioning, muscle strengthening and increasing joint flexibility activities. Aerobic conditioning consisted of walking on a treadmill for 20 to 40 minutes per session. Exercise intensity was controlled based on the individual pre-programmed target heart rate (50\% of work in the maximal heart rate) and was monitored using a heart rate monitor (Polar Accurex Plus, Lake Success, NY, USA). The target heart rate was calculated by the Karvonem equation: \% of work $\mathrm{x}$ (maximal heart rate - baseline heart rate) + baseline heart rate, corrected for resting heart rate on the day of exercise. ${ }^{17}$ Thus, strain intensity (speed, in $\mathrm{km} / \mathrm{h}$ ) was gradually adjusted to maintain the target heart rate. For skeletal muscle strengthening, local exercises consisting of flexion and extension of the lower and upper limbs were implemented, with body mass-imposed resistance or external resistance using dumbbells and bars. Relaxation and elongation exercises were conducted before and after every session, including stretching to increase the flexibility of large muscle-joint groups (shoulder, elbow, hip joint, knee and ankle).

\section{Functional Capacity Assessment}

Aerobic resistance was evaluated by walking 800 meters, according to the recommendations described by Andreotti \& Okuma. ${ }^{18}$ Muscle strength was evaluated by standardized tests according to Rikli \& Jones. ${ }^{19}$ Sitting down and standing up from a chair (lower limb component of body strength) and elbow flexion (upper limb component of body strength) were analyzed using the number of repetitions in 30 seconds for evaluation of the strength. Agility and flexibility levels were 
determined by performance during previously described tests. ${ }^{18,20}$ Agility was evaluated by the time it took to stand up from a chair and from the floor, and flexibility was evaluated by the sit and reach test.

\section{Quality of Life Assessment}

Quality of life was evaluated by a shortened version of the WHOQOL questionnaire, ${ }^{21}$ validated for a Brazilian population. ${ }^{22}$ The questionnaire contained 26 questions providing information about several factors of quality of life, including: the Physical Domain, relating to pain or discomfort, energy or fatigue, sleep, rest, mobility, daily activities, medicine dependency, and job performance; the Psychological Domain, relating to feelings, learning, memory and attention, self-esteem, aspect, spirituality, religiousness, and positive or negative thinking; the Social Domain, concerning personal relationships, social support and sexuality; and finally, the Environmental Domain, relating to physical security, home environment, financial security, opportunity for information assessment, event participation and activities undertaken during spare time. Each domain was scaled from zero to 100 points, and higher scores represented a better quality of life.

\section{Statistical Analysis}

Data are presented as mean \pm standard deviation (SD). Two-way analysis of variance (ANOVA) was used to determine differences between groups before and after training, followed by Bonferroni post hoc tests. Comparisons of changes in variables between groups were performed using unpaired Student's t-tests. Statistical significance was established at $\mathrm{p}<0.05$.

\section{RESULTS}

During the study period, 3 patients from the untrained group experienced an impairment of symptoms and were hospitalized. At the conclusion of the protocol, 2 others could not be found for outcome acquisition and their data were excluded from analysis. In the trained group, 2 patients dropped out of the study and 4 patients did not complete a minimum of $80 \%$ of the exercise program. Consequently, our results are based on data from 42 patients $(S: n=20 ; T$ : $\mathrm{n}=22$ ). For the $\mathrm{T}$ group, the mean frequency of the exercise program was $89 \pm 4 \%$.

Table 1 shows the general characteristics, heart failure etiology and pharmacological therapy of the subjects. Different etiologies were identified in patients including myocardial infarction, systemic hypertension, dilated
Table 1 - General and clinical characteristics of subjects in the study

\begin{tabular}{lcc}
\hline Parameters & $\begin{array}{c}\text { Untrained } \\
(\mathrm{n}=20)\end{array}$ & $\begin{array}{c}\text { Trained } \\
(\mathrm{n}=22)\end{array}$ \\
\hline Age (years) & $60 \pm 11$ & $61 \pm 12$ \\
Gender (male/female) & $18 / 2$ & $19 / 3$ \\
Heart Failure etiology (\%) & & \\
$\quad$ Myocardial infarction & $10(50 \%)$ & $9(45 \%)$ \\
$\quad$ Systemic hypertension & $4(20 \%)$ & $4(18 \%)$ \\
$\quad$ Dilated Chagas' cardiomyopathy & $3(15 \%)$ & $2(9 \%)$ \\
$\quad$ Diabetes mellitus & $1(5 \%)$ & $1(4 \%)$ \\
Medication (\%) & $20(100 \%)$ & $22(100 \%)$ \\
$\quad$ Beta-blockers & $18(80 \%)$ & $20(91 \%)$ \\
Diuretics & $20(100 \%)$ & $22(100 \%)$ \\
ACE inhibitors & $10(50 \%)$ & $15(68 \%)$ \\
$\quad$ Digitalis &
\end{tabular}

cardiomyopathy and diabetes mellitus. During the followup, medicine doses were not modified except for those that presented impairment of symptoms and, consequently, these patients were excluded from our analysis.

At the beginning of the study, and before the training program began, there were no significant differences between groups $\mathrm{S}$ and $\mathrm{T}$ for any functional capacity variables (Figure 1). However, after 6 months of follow-up, the $T$ group had an impressive enhancement of performance on all functional tests (Figure 1). Furthermore, in trained patients, aerobic capacity was significantly improved, with the time to walk 800 meters reduced by $33 \pm 8 \%$ (before: $16 \pm 0.5$ vs. after: $12 \pm 0.9$ minutes, $\mathrm{p}<0.01$ ) whereas no significant difference was found in untrained patients (before: $16.5 \pm 0.8$ vs. after: $15 \pm 0.9$ minutes). After the training period, skeletal muscle strength was improved in the $\mathrm{T}$ group, evaluated by the sitting and standing up from the chair test (S: $5 \pm 3 \%$ vs. T: $47 \pm 10 \%$ improvement; $\mathrm{p}<0.001)$ and arm flexion (S: $1 \pm$ 1 vs. T: $26 \pm 3 \%$ improvement; $\mathrm{p}<0.001)$. Tests analyzing the time taken to stand up from a chair and to stand up from the floor showed a significant reduction $(\mathrm{p}<0.001)$ in the $\mathrm{T}$ group (chair: $-39 \pm 4 \%$ and floor: $-44 \pm 9 \%$ change in time) compared to the S group (chair: $0.8 \pm 0.2 \%$ and floor: $4 \pm$ $2 \%$ change in time), indicating an improvement in the agility of patients submitted to physical exercise training. Patients from the $\mathrm{T}$ group also had a significant enhancement $(\mathrm{p}<$ $0.001)$ of joint flexibility ( $46 \pm 6 \%$ improvement) when compared to the untrained group ( $6 \pm 6 \%$ improvement).

After 6 months of follow-up, there was some general improvement in the parameters of quality of life, but significant statistical differences were only noted for the 
exercised patients (Figure 2). All domains, including physical (S: $2 \pm 1$ vs. T: $23 \pm 4 \%$ improvement), psychological (S: 1 \pm 1 vs. T: $20 \pm 2 \%$ improvement), social (S: $3 \pm 2$ vs. T: 16 $\pm 1 \%$ improvement) and environmental (S: $2 \pm 1$ vs. T: 15
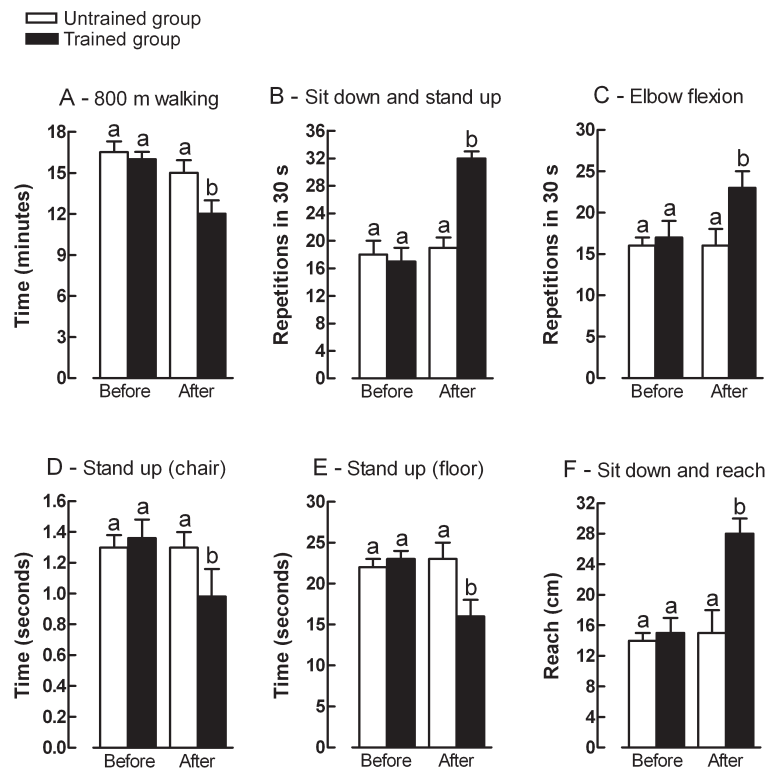

Figure 1 - Functional capacity before, and 6 months after, physical exercise evaluated by fitness tests involving aerobic conditioning (Panel A); strength of lower (Panel B) and upper limbs (Panel C); agility (Panels D and E); and joint flexibility (Panel F). Different letters indicate statistically significant differences between the groups $(\mathrm{p}<0.05)$. Data are expressed as the mean $\pm \mathrm{SD}$
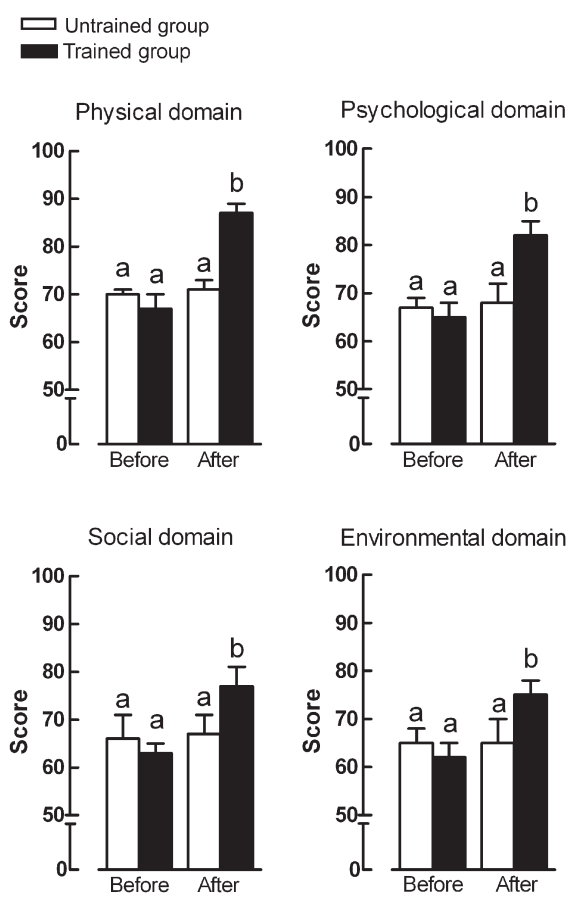

Figure 2 - Quality of life evaluated by the multiple domain questionnaires both before and after 6 months of physical exercise. Different letters indicate statistically significant differences between the groups $(\mathrm{p}<0.001)$. Data are expressed as the mean $\pm \mathrm{SD}$ $\pm 2 \%$ improvement), were significantly improved in the $\mathrm{T}$ group $(\mathrm{p}<0.001)$ in comparison to the $\mathrm{S}$ group.

\section{DISCUSSION}

Heart failure plays an important role in public health, being one of the major complications of heart disease and a leading cause of death in developed Western countries. It may also pose a significant economic burden for health budgets. $^{2}$ The natural history of this illness constitutes impairment of functional capacity, physical performance, and ability to perform daily activities, finally becoming detrimental to the quality of life. ${ }^{2,23}$ For these reasons, therapeutic approaches that substantially improve these aspects greatly favor a better prognosis. ${ }^{24}$

Previous studies furnished data concerning exercise as a prescription for patients with heart failure, ${ }^{14-16,25}$ but were performed on specially selected subjects who were comparatively young, mostly male, and had an ischemic etiology. Thus, the important contribution of this present work was to demonstrate the beneficial role of physical exercise for the improvement of functional capacity, quality of life and general sense of well-being. Additionally, there were no indications of any harmful effects of these training protocols, which corroborates previous studies ${ }^{8,26,27}$ that also demonstrated the safety of physical exercise in rehabilitation programs.

It has been shown that supervised exercise for 3 to 6 months may enhance peak oxygen consumption by $11 \%$ to $36 \% .{ }^{28}$ In our study, although oxygen consumption was not determined, trained patients had a notable enhancement of performance in the 800-meter walking test, suggesting an improvement in aerobic condition. This improvement in aerobic fitness may be the basis for several beneficial physiological changes in the patients, including reductions in maximal heart rate, systolic blood pressure, and oxygen consumption required by the myocardium during activities of moderate and high intensity. ${ }^{25}$ In addition, the improvement in performance during effort testing may also lead to an expectation of a lower incidence of fatal and non-fatal events in patients with heart failure. ${ }^{25}$

Researchers have reported that the ability to exercise perhaps not only depends on the degree of cardiac dysfunction established, ${ }^{8,23}$ but that changes in the skeletal muscles may also play a relevant role, leading to significant reduction of muscle strength and intensification of weakness and fitness in patients with heart failure compared to a matched healthy population. ${ }^{29,30}$ In the present work, we observed significant improvements in muscle strength, agility and joint flexibility resulting from a supervised physical exercise program with relatively short duration. The 
improvement of functional capacity and fitness in the trained group of patients suggests a safety basis for the execution of daily and routine activities and, additionally, a diminution of falls, bone fractures and disabling symptoms such as fatigue and dyspnea. ${ }^{26}$

The trinity of physical exercise, functional capacity and quality of life in patients with heart failure has already been studied in clinical trials. ${ }^{25,26,31}$ Therefore, with the fundamental aim of evaluating whether physical exercise practice can improve social and personal well-being and quality of life, we applied a validated questionnaire for patients with heart failure. From this questionnaire, we noted a significant enhancement in all parameters of quality of life in exercised patients, including physical, psychological, social and environmental domains as shown in Figure 2. These findings agree with others investigators ${ }^{30}$ and assume an important context, since studies have demonstrated a strong association between recurrent cardiovascular events and psychosocial problems such as depression, anxiety and social isolation. ${ }^{25,32}$

In conclusion, a supervised and guided physical exercise program is safe and beneficial for patients with heart failure with different etiologies. Our data also support the concept that exercise must be part of a heart failure treatment plan, thereby significantly improving fitness, functional capacity to carry out tasks of daily living, independence, psychosocial well-being, and consequently, the quality of life of these patients.

\section{Limitations of the study}

In the present study, two main limitations must be considered. The exercise intensity was based on heart rate peak acquired by the ergometric test. The rationale for using heart rate for guiding exercise intensity is based on the relatively linear relationship between heart rate and $\mathrm{VO}_{2}$ in exercise training programs. ${ }^{33}$ However, an exercise training prescription based only on heart rate peak has been shown to overestimate exercise intensity. ${ }^{34}$ In addition, although the $\mathrm{VO}_{2}$ peak is often considered the gold standard parameter for assessment of functional capacity, we used tests and physical evaluations based on tasks of daily living according to a method standardized for elderly people. ${ }^{17}$

\section{ACKNOWLEDGMENT}

Special acknowledgment is given to Mr. Charles G. Rees, for assistance with language correction and style.

\section{REFERENCES}

1. Sociedade Brasileira de Cardiologia. Diretriz latino-americana para avaliação e conduta na insuficiência cardíaca descondensada. Arquivos Brasileiros de Cardiologia. 2005;85:1-48.

2. Hunt SA, Abraham WT, Chin MH, Feldman AM, Francis GS, Ganiats T G, et al. ACC/AHA 2005 Guideline Pdate for the Diagnosis and Management of Chronic Heart Failure in the Adult A Report of the American College of Cardiology/American Heart Association Task Force on Practice Guidelines (Writing Committee to Update the 2001 Guidelines for the Evaluation and Management of Heart Failure). Circulation. 2005;112:154-235.

3. Pescatello LS, Di Pietro L. Physical Activity in Older Adults: An Overview of Health Benefits. Sports Medicine. 1993;15:353-64.

4. Wielenga RP, Erdman R, Huisveld IA, Bol E, Dunselman PH, Baselier MR. Effect of Exercise Training on Quality of Life in Patients with Chronic Heart Failure. Journal Psychosom Research. 1998;45:45964.

5. Dubach P, Sixt S, Meyers J. Exercise Training in Chronic Heart Failure: Why, When and How. Swiss Medical Weekly. 2001;13:510-14.

6. Cowie MR, Zaphirou A. Management of Chronic Heart Failure. British Medical Journal. 2002;325:422-25.

7. Sullivan MJ, Higginbotham MB, Cobb FR. Exercise Training in Patients with Chronic Heart Failure Delays Ventilatory Anaerobic Threshold and Improves Submaximal Exercise Performance. Circulation. 1989:79,32429.
8. Willenheimer R, Erhardt L, Cline C, Rydberg E, Isrelsson B. Exercise Training in Heart Failure Improves Quality of Life and Exercise Capacity. European Heart Journal. 1998;19,774-81.

9. Maiorana A, O'Driscoll G, Cheetham C, Collis J, Goodam C, Rankin $\mathrm{S}$, et al. Combined Aerobic and Resistance Exercise Training Improves Functional Capacity and Strength in CHF. Journal of Applied Physiology. 2002;88:1565-70.

10. Shephard RJ. Physiological Basis of Training in the Elderly. Science \& Sports. 1994;9:189-96.

11. Bellardinelli R, Georgiou D, Cianci G, Purcaro A. Randomized, Controlled Trial of Long-Term Moderate Exercise Training in Chronic Heart Failure. Circulation. 1999;99:1173-82.

12. Willenheimer R, Rydberg E, Cline C, Broms K, Hillberger B, Oberg L, et al. Effects on Quality of Life, Symptoms, and Daily Activity 6 months after Termination of an Exercise Training Programme in Heart Failure Patients. International Journal of Cardiology. 2001;77:25-31.

13. Jetté M, Heller R, Landry F, Blumchen G.: Randomized 4-week Exercise Program in Patients with Impaired Left Ventricular Function. Circulation. 1991;84:1561-7.

14. Piña IL, Apstein CS, Balady GJ, Belardinelli R, Chaitman BR, Duscha BD, et al. Exercise and Heart Failure: A Statement from the American Heart Association Committee on Exercise, Rehabilitation and Prevention. Circulation. 2003;107:1210-25. 
15. II Diretrizes da Sociedade Brasileira de Cardiologia sobre teste ergométrico. Arquivos Brasileiros de Cardiologia. 2002;78:Suppl II:118.

16. Swedberg K, Cleland J, Dargie H, Drexler H, Follath F, Komajda M, et al. Guidelines for the Diagnosis and Treatment of Chronic Heart Failure: Executive Summary: The Task Force for the Diagnosis and Treatment of Chronic Heart Failure of the European Society of Cardiology. European Heart Journal. 2005;26:1115-40.

17. Silva MSV, Bocchi ED, Guimarães GV, Padovani CV, Silva MHG, Pereira SF, Fontes, FD. Benefício do Treinamento Físico no Tratamento da Insuficiência Cardíaca. Estudo com Grupo Controle. Arquivos Brasileiros Cardiologia. 2002;79:351-6.

18. Andreotti RA, Okuma SS. Validação de uma Bateria de Testes de Atividade da Vida Diária para Idosos Fisicamente Independentes. Revista Paulista de Educação Física. 1999;13:46-66.

19. Rikli R;Jones J. Development and Validation of a Functional Fitness Test for Community-Residing Older Adults. Journal of Aging and Physical Activity. 1999;7:129-61.

20. Stanziola L, Prado JF. Medidas de agilidade. In: Matsudo V, editor. Testes em Ciências do Esporte. São Caetano do Sul: Celafiscs. 1995;73-7.

21. WHOQOL Group. The World Health Organization Quality of Life Assessment (WHOQOL): Development and General Psychometric Properties. Social Science and Medicine. 1998;46:1569-85.

22. Fleck MPA, Louzada S, Xavier M. Aplicação da Versão em Português do Instrumento Abreviado de Avaliação da Qualidade de Vida "WHOQOLBref”. Revista de Saúde Pública. 2000;34:178-83.

23. McKelvie RS, Teo KK, McCartney N, Humen D, Montague T, Yusuf S. Effects of Exercise Training in Patients with Congestive Heart Failure: A Critical Review. Journal of American College Cardiology. 1995;25:78996.

24. Keteyian SJ, Kraus WE. Introduction: exercise in patients with chronic heart failure. Heart Failure Reviews. 2008;13,1-2.

25. Leon AS, Franklin BA, Costa F, Balady GJ, Berra KA, Stewart KJ, et al. Cardiac Rehabilitation and Secondary Prevention of Coronary Heart Disease: An American Heart Association Scientific Statement From the Council on Clinical Cardiology (Subcommittee on Exercise, Cardiac Rehabilitation, and Prevention) and the Council on Nutrition, Physical Activity, and Metabolism (Subcommittee on Physical Activity), in Collaboration With the American Association of Cardiovascular and Pulmonary Rehabilitation. Circulation. 2005;111:369-76.
26. Coats AJS, Adamopoulos S, Radaelli A, McCance A, Meyer TE, Bernardi L, et al. Controlled Trial of Physical Training in Chronic Heart Failure. Exercise Performance, Hemodynamics, Ventilation and Autonomic Function. Circulation. 1992;85:2119-31.

27. McKelvie RS. Exercise training in patients with heart failure: clinical outcomes, safety, and indications. Heart Failure Reviews. 2008;13,311.

28. Wenger NK, Froelicher ES, Smith LK, Ades PA, Berra K, Blumenthal JA, et al. Cardiac rehabilitation as secondary prevention. Agency for Health Care Policy and Research and National Heart, Lung, and Blood Institute. Clinical Practice Guidelines Quick References Guidelines Clinics. 1995; 17:1-23.

29. Lipkin DP, Jones DA, Round JM, Poole-Wilson PA. Abnormalities of Skeletal Muscle in Patients with Chronic Congestive Heart Failure. International Journal of Cardiology. 1988;18:187-95.

30. Buller NP, Jones D, Poole-Wilson PA. Direct Measurement of Skeletal Muscle Fatigue in Patients with Chronic Heart Failure. British Heart Journal. 1991;65:20-4.

31. Lavie CJ, Milani RV. Effects of Cardiac Rehabilitation Programs on Exercise Capacity, Coronary Risk Factors, Behavioral Characteristics, and Quality of Life in a Large Elderly Cohort. American Journal of Cardiology. 1995;76:177-79.

32. Rozanski A, Blumenthal JA, Kaplan J. Impact of Psychological Factors on the Pathogenesis of Cardiovascular Disease and Implications for Therapy. Circulation. 1999;99:2192-2217.

33. Working Group on Cardiac Rehabilitation and Exercise Physiology and Working group on Heart Failure of the European Society of Cardiology. Recommendations for exercise testing in chronic heart failure patients. Eur Heart J. 2001;22:37-45.

34. Rondon MU, Forjaz CLM, Nunes N, Amaral SL, Barretto ACP, Negrão CE. Comparação entre a Prescrição de Intensidade de Treinamento Físico Baseada na Avaliação Ergométrica Convencional e na Ergoespirométrica. Arq Bras Cardiol. 1998;70:159-66. 